\title{
High-speed optical recording in vitreous chalcogenide thin films
}

\author{
A.A. Kryuchyn ${ }^{1}$, V.V. Petrov ${ }^{1}$, V.M. Rubish ${ }^{2}$, A.S. Lapchuk ${ }^{1}$, S.O. Kostyukevych ${ }^{3}$, \\ P.E. Shepeliavyi ${ }^{3}$, K.V. Kostyukevych ${ }^{3}$ \\ ${ }^{1}$ Institute for Information Recording, NAS of Ukraine \\ 2, Shpak str., 03113 Kyiv, Ukraine \\ E-mail: petrov@ipri.kiev.ua \\ ${ }^{2}$ Uzhgorod Scientific-Technological Center of the Institute for Information Recording, NAS of Ukraine, \\ 4a, Zamkovi skhody str., 88000 Uzhgorod, Ukraine, \\ E-mail: center.uzh@gmail.com \\ ${ }^{3}$ V. Lashkaryov Institute of Semiconductor Physics, NAS of Ukraine \\ 41, prospect Nauky, 03028, Kyiv, Ukraine \\ E-mail:sergeyk@isp.kiev.ua
}

\begin{abstract}
Thin films of glassy chalcogenide semiconductor are widely used as recording media in optical data storage. To obtain relief micro- and nanoscale structures on the surface of optical master discs inorganic photoresists based on chalcogenide glassy semiconductors can be used. They have high resolution and allow for exposure by short laser pulses. Implementation of such exposure is promoted by increasing the speed of photostructural transformations at high powers of exposing radiation. This increase in the sensitivity is associated with both local heating by illumination and a high density of excited electron-hole pairs. The exposure mode of the inorganic photoresists based on glassy chalcogenide semiconductor pulses of $10^{-8}-10^{-9} \mathrm{~s}$ is close to the threshold of local photothermal destruction. Significant impact on the value of the threshold of photothermal destruction effects the choice of the substrate material which determines the rate of heat removal from the irradiation area. Moreover, one also needs to consider the effect of pulsed annealing of the inorganic photoresist material on the process of selective etching. We have established an inversion of the selective etching of the inorganic negative photoresist based on $\mathrm{As}_{2} \mathrm{~S}_{3}$ in the center of the irradiated zone. The diameter of this zone is about $20 \%$ of the diameter of exposing beam.

After the selective etching in alkaline solution in the center of protrusions being formed on the substrate, there observed are some dimples with the depth of $30-50 \mathrm{~nm}$. Prior to the processing of irradiated inorganic photoresist by the selective etching these dimples were absent and their appearance is not due to possible local material evaporation of the inorganic photoresist. A possible reason for the inversion of solubility of the inorganic photoresist could be pulsed annealing in the recording process.
\end{abstract}

Keywords: inorganic photoresists, laser exposure, selective etching, inversion of solubility.

Manuscript received 18.02.14; revised version received 05.08.14; accepted for publication 29.10.14; published online 10.11.14.

\section{Introduction}

Inorganic photoresists, as shown by numerous experiments [1-4], can be used to record information on the master discs (both CD and DVD). Currently, it is of interest to use writing data pulses $(10-100) \mathrm{ns}$ on the master disks at a rate of tens of millions of bits per second onto carriers that move relatively to the recording beam having linear velocity of $6-12 \mathrm{~m} / \mathrm{s}$. The use of inorganic photoresists for high-speed mastering promotes an increased sensitivity at high power densities of output exposure radiation, which is associated with both local heating in the radiation zone and the generation of a high density of electron-hole pairs $[3,5,6]$.

As demonstrated by studies being conducted in recent years, a significant impact on the performance of the inorganic photoresists is provided by the thermal treatment mode [7]. 


\section{Experimental}

The light exposure of inorganic photoresists was carried out at the laser writing station described in detail in [8]. Distinctive features of the process of pulsed laser exposure consist in the fact that, in order to reduce the size of prints, the ultraviolet semiconductor laser with the wavelength $405 \mathrm{~nm}$ is used, and to improve both the accuracy and speed of the auto focus system actuator a piezoelectric mechanism for moving the microscope is applied [9].

In the process of laser writing, the master disc was manufactured in the following manner: Ø120 mm glass substrates were covered with a chalcogenide semiconductor film having the thickness 180 to $200 \mathrm{~nm}$ by using the thermal vacuum deposition. In the experiments, chalcogenide semiconductor compounds $\mathrm{As}_{2} \mathrm{~S}_{3}$ and As-S-Se were used.

To obtain the relief micro-images on the inorganic photoresists, we used some sort of anhydrous selective etchants based on amines [10]. Shape control of the formed structure was provided by an atomic force microscope.

The accomplished experimental tests have shown that at high power densities for the negative inorganic resist $\left(\mathrm{As}_{2} \mathrm{~S}_{3}\right)$ solubility inversion was observed in the center of irradiated zone (Fig. 1). Immediately after the exposure of the inorganic photoresist films, there were no signs of the periodic micro-relief structure. The surface of the photoresist was mirror-reflective. A diffraction pattern was obtained only after the selective etching of the photoresist. This indicates that the recording process does not provide the photoresists' local photo-thermal destruction.

The performed experiments concerning information recording with a focused laser radiation of the $405-\mathrm{nm}$ wavelength (aperture of the focusing objective was 0.85 ) and optimum power showed that the obtained width of prints lies within the range 0.1 to $0.3 \mu \mathrm{m}$. The latter values depended on the power of exposing radiation. The typical view of the master disc surface after processing the non-organic resist layer in selective etchant and its transverse section are shown in Fig. 2.

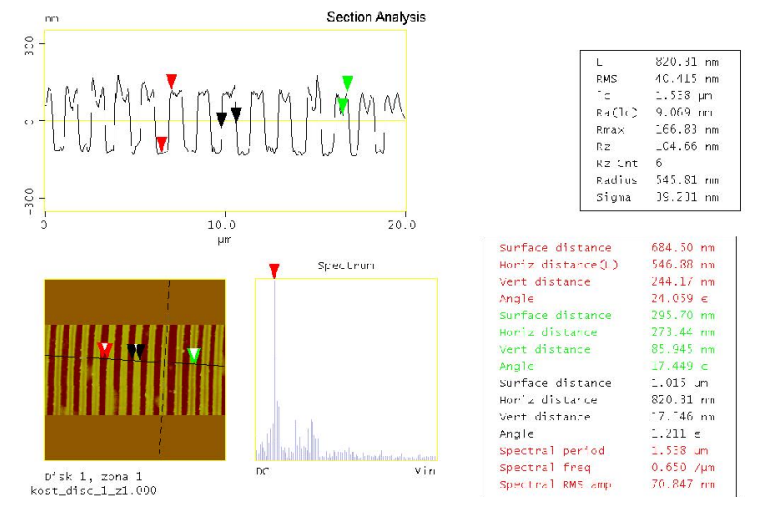

Fig. 1. The relief microstructure on the negative inorganic resist $\mathrm{As}_{2} \mathrm{~S}_{3}$.

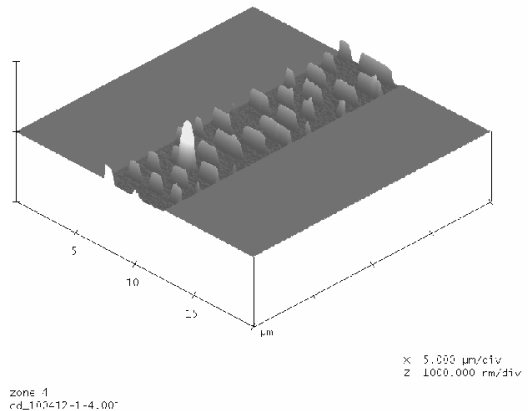

a)

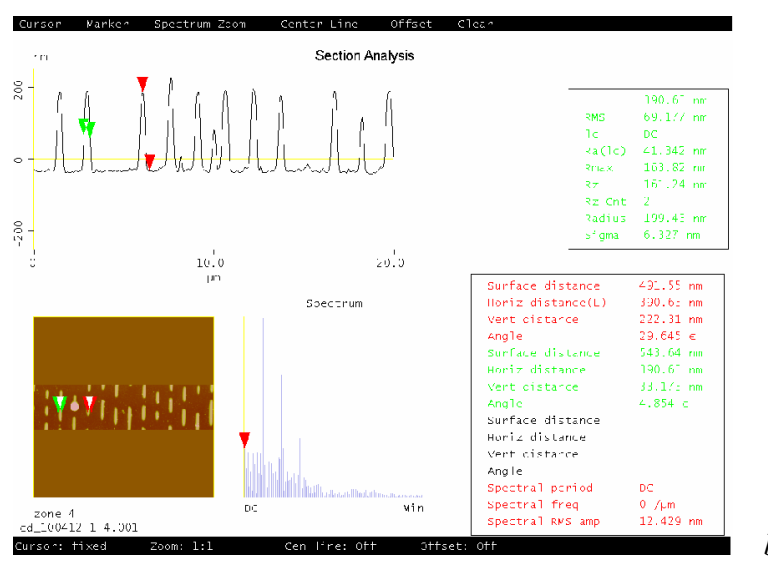

b)

Fig. 2. Micro-relief of the image recorded in the layer of negative non-organic photoresist after exposing the layer with laser radiation $1 \mathrm{~mW}$ power at the micro-objective output. a) general view of the master disc surface; b) transverse section of the recorded pits.

To create combined elements, we performed twofold exposing the negative resist with various powers of laser radiation. General view of the master disc surface after two-fold exposing with various powers and the following processing the layer in selective etchant as well as respective transverse section of the prints are shown in Fig. 3.

The obtained results allows to conclude that these non-organic resists enable to create various combined elements with various pit heights, including multi-level discs. When choosing the recording modes, a special attention should be paid to exposing the pits with the minimum length. Using the recording system with a high resolution, one can decrease the size of recorded prints by $30 \%$.

\section{Discussion of the results}

For the analysis of the experimental results, we carried out numerical simulation of heating the film of inorganic photoresist. 


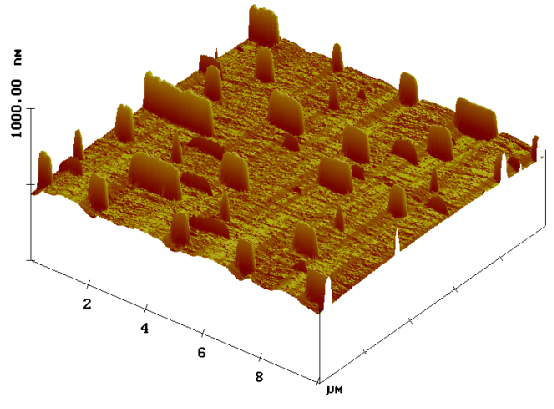

a)
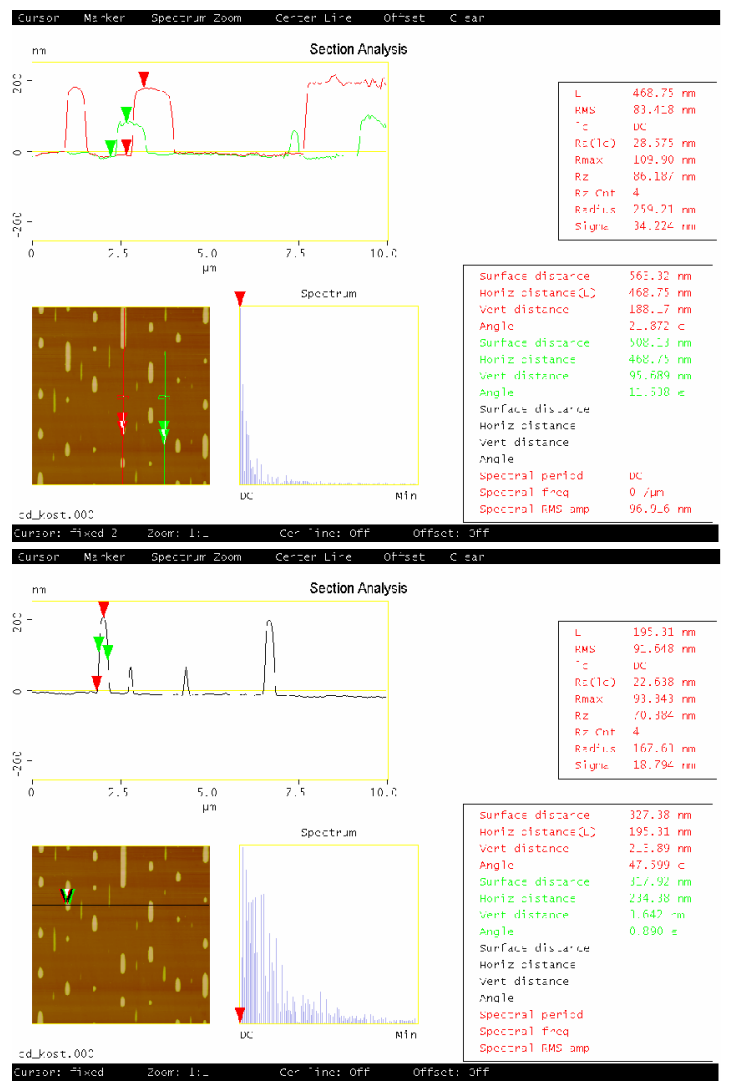

b)

Fig. 3. Micro-relief of the image recorded in the layer of negative non-organic photoresist after two-fold exposing with various powers of laser radiation: a) general view of the master disc surface; b) transverse section of recorded pits along the direction of recording; c) transverse section of recorded pits along the normal to the direction of recording.

The temperature distribution dynamics inside solid is described by differential diffusion equation

$$
\frac{\partial T}{\partial t}=\frac{k}{c_{p} \rho}\left(\frac{\partial^{2} T}{\partial x^{2}}+\frac{\partial^{2} T}{\partial y^{2}}+\frac{\partial^{2} T}{\partial z^{2}}\right)+\frac{q}{c_{p} \rho} .
$$

Here $c_{p}, \rho, k$ are heat capacity, specific gravity and thermal conductivity of solid, $q$ is the specific heat power distributed in the volume of solid, the ratio $\alpha=\frac{k}{c_{p} \rho}$ is the thermo-diffusion factor. Under the light irradiation, the distributed power of heat sources is determined by the light intensity and absorption coefficient $\eta$ of light by the following formula

$$
q=|\vec{E}(x, y, z)|^{2} \eta=I(x, y, z) \eta \text {. }
$$

Our simulation was performed on the basis of the mathematical apparatus of the Green function. It was assumed that the thermoresist is thick and all the energy absorption takes place inside the thermoresist layer and far from its substrate. Therefore, the substrate can be replaced by medium with the same thermal characteristics as thermoresist and the layer have, but which does not absorb the electromagnetic energy. The model also ignored the thermoresist thermal reaction with air and the thermoresist temperature intended to be uniform when reacting with air. Under this approximation, the change of the temperature distribution in the thermoresist can be written as

$\Delta T(z, r, t)=\frac{\eta}{c_{p} \rho_{0}} \int_{0}^{t} d t^{\prime} \int G\left(z, r, t, z^{\prime}, r^{\prime}, t^{\prime}\right) \times$

$\times I\left(z^{\prime}, r^{\prime}, t^{\prime}\right) r^{\prime} d r^{\prime} d \varphi^{\prime} d z^{\prime}$,

where

$$
\begin{aligned}
& G\left(z, r, t, z^{\prime}, \rho^{\prime}, t^{\prime}\right)= \\
& =\frac{1}{\left(4 \pi \alpha\left(t-t^{\prime}\right)\right)^{3 / 2}} \exp \left(-\frac{r^{2}+r^{\prime 2}-2 r r^{\prime} \cos \left(\varphi-\varphi^{\prime}\right)}{4 \alpha\left(t-t^{\prime}\right)}\right) \times \\
& \times\left(\exp \left(-\frac{\left(z-z^{\prime}\right)^{2}}{4 \alpha\left(t-t^{\prime}\right)}\right)+\exp \left(-\frac{\left(z+z^{\prime}\right)^{2}}{4 \alpha\left(t-t^{\prime}\right)}\right)\right)
\end{aligned}
$$

is Green function of the diffusion equation for the half of space limited by insulating wall (see Fig. 4). Having provided the necessary integration over the coordinate $\varphi^{\prime}$ by using the well-known formula

$$
\int_{0}^{\pi} \exp (x \cdot \cos (\theta)) \cos (n x) d x=\pi I_{n}(x)[11],
$$

Eq. (3) for temperature distribution in the thermoresist is reduced to

$$
\begin{aligned}
& \Delta T(r, \varphi, z, t)=\frac{\eta}{c_{p} \rho} \\
& I_{0} \exp \left(-r^{\prime 2} / R^{2}\right) \exp \left(-\eta z^{\prime}\right) \theta\left(t^{\prime}\right) \theta\left(\tau-t^{\prime}\right) \\
& \int_{0}^{t} \frac{2 \pi}{\left(4 \pi k\left(t-t^{\prime}\right)\right)^{3 / 2}} d t^{\prime} \times \\
& \times \int_{0}\left(\frac{2 r r^{\prime}}{4 \alpha\left(t-t^{\prime}\right)}\right) \exp \left(-\frac{r^{2}+r^{\prime 2}}{\alpha k\left(t-t^{\prime}\right)}\right) \\
& \left.\quad \exp \left(-\frac{\left(z-z^{\prime}\right)^{2}}{4 \alpha\left(t-t^{\prime}\right)}\right)+\exp \left(-\frac{\left(z+z^{\prime}\right)^{2}}{4 \alpha\left(t-t^{\prime}\right)}\right)\right) \rho^{\prime} d \rho^{\prime} d z^{\prime}
\end{aligned}
$$


where $\tau$ is the duration of the light pulse. Used in the formula (5) is the Gauss model of a focused laser beam with an intensity distribution being calculated using the formula

$$
I(x, y, z)=I_{0} \exp \left(-\frac{x^{2}+y^{2}}{R^{2}}\right) \exp (-\eta z) .
$$

When the recording mode is switched on, significant local heating of the resist is observed inside the irradiation zone of inorganic photoresist. That is seen from Figs 5 and 6 depicting the radial temperature distribution inside the radiation zone. In our simulations, the focused Gaussian blue laser beam diameter was $500 \mathrm{~nm}$ (that is the same as in BluRay disc). The following physical parameters for $\mathrm{As}_{2} \mathrm{~S}_{3}$ were used in calculations: $\left(c_{p}=470 \mathrm{~J} / \mathrm{kg} \mathrm{K} ; \rho=3330 \mathrm{~kg} / \mathrm{m}^{3} ; \alpha=\right.$ $\left.8.3 \cdot 10^{-8} \mathrm{~m}^{2} / \mathrm{s} ; n^{\prime}=0.318\right)$.

Simulation of heating the inorganic photoresist shows that the center of illuminated area has a maximum temperature of heating the photoresist, the value of which decreases rapidly with increasing the distance from the center of irradiation (Fig. 5).

The heating temperature of the photoresist decreases rapidly with the depth inside the photoresist (Fig. 6). Substantially more surface layers are heated in the center of the irradiated area. This fact may explain the inverse solubility in this region.

As it stems from the demonstrated images of the relief microstructures (Fig. 1), the inversion of solubility is observed only in the center of the irradiation zone at a certain temperature. At the edges of the relief microstructures and in the areas with a lower exposing power, this effect is not revealed. Due to the fact that formation of hollows in elements of the relief microstructures is observed in photoresists of different composition, one may assume that the revealed effect is mainly thermal. Previously, the effect of inversion selective dissolution of the negative photoresist was observed under irradiation and selective etching of the inorganic resists annealed at a temperature close to the softening temperature [7]. A similar effect was found in our experiments, in which the inorganic photoresist exposure was carried out at elevated photoresist temperatures.

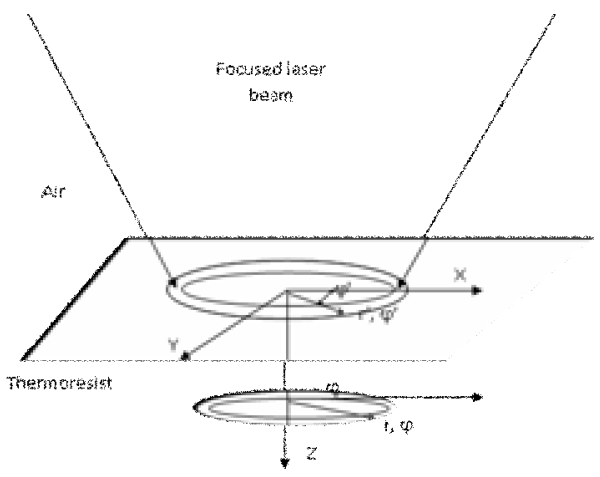

Fig. 4. Geometry of the problem.

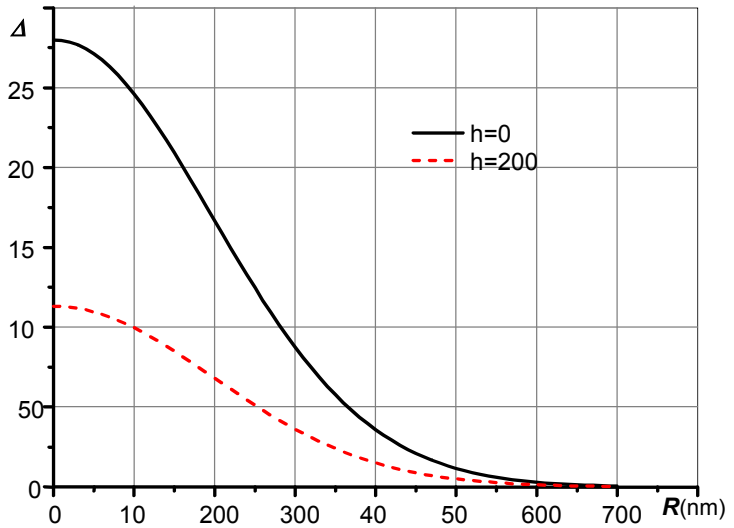

(a) $\Delta t=100 \mathrm{~ns} ; P=0.5 \mathrm{~mW}$

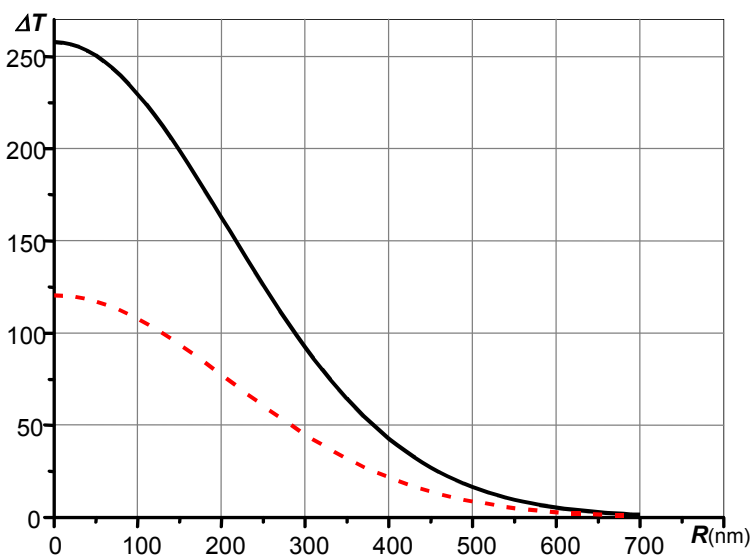

(b) $\Delta t=200 \mathrm{~ns} ; P=0.3 \mathrm{~mW}$

Fig. 5. Radial dependence of temperature changes apart from the center of light spot on the upper (solid line) and lower (dashed line) surfaces of the semiconductor photoresist under different powers and duration of the laser radiation pulses: $H=$ $200 \mathrm{~nm}$;

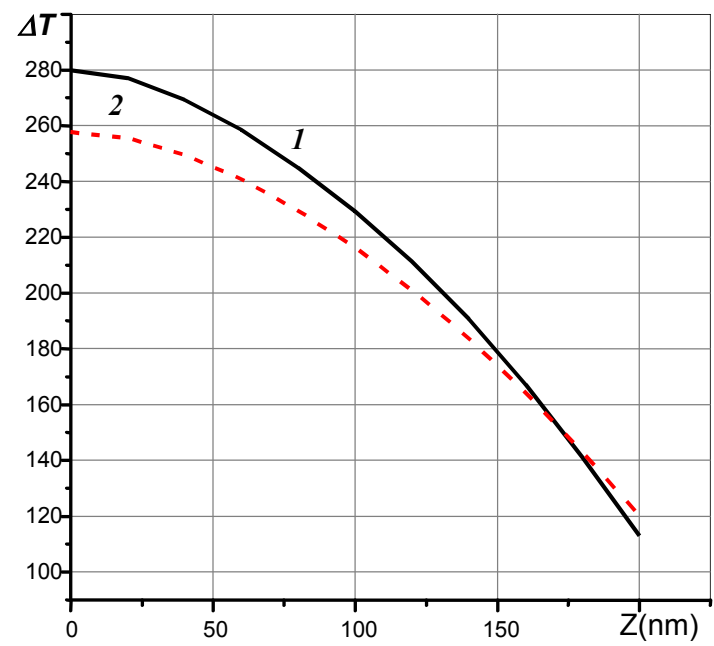

Fig. 6. Radial distribution of the temperature increase through the semiconductor photoresist thickness, $h=200 \mathrm{~nm} ; 1-\mathrm{P}=$ $0.5 \mathrm{~mW} ; \Delta t=100 \mathrm{~ns}, 2-P=0.3 \mathrm{~mW} ; \Delta t=200 \mathrm{~ns}$ (at the upper surface of registering medium). 
This effect has to be considered after exposure of negative inorganic photoresists. The size of hollows on the relief micro-formations is tens of nanometers, and under certain conditions the negative inverse solubility of inorganic resists can be used to produce nano-sized structures.

\section{Conclusions}

1. It has been found the effect of solubility inversion for negative inorganic photoresists when pulsed laser exposure is being applied. The effect was observed in inorganic photoresists having various compositions $\left(\mathrm{As}_{2} \mathrm{~S}_{3}\right.$ and $\left.\mathrm{As}-\mathrm{S}-\mathrm{Se}\right)$.

2. The observed effect may be caused by local heating the photoresist above the threshold temperature at the center of irradiated zone.

\section{Acknowledgments}

The authors would like to express their sincere gratitude to Yu.A. Borodin, scientific researcher of the Institute for Information Recording, NAS of Ukraine, for recording information on master discs; V.I. Min'ko, senior scientific researcher of the Institute of Semiconductor Physics, NAS of Ukraine, for vacuum deposition of the registering media; and A.A. Kudryavtsev, senior scientific researcher of the Institute of Semiconductor Physics, NAS of Ukraine, for taking part in discussion of paper materials and editing it.

\section{References}

1. V.V. Petrov, A.A. Kryuchyn, S.A. Kostyukevich, V.M. Rubish, Inorganic Photolitography. G. Kyrdiumov Institute for Metal Physics, National Academy of Sciences, Kyiv, p. 196, 2007 (in Ukrainian).

2. S.O. Kostyukevich, I.Z. Indutny, P.E. Shepelyavii, Laser writing of relief microstructures on balls $\mathrm{As}_{40} \mathrm{~S}_{40} \mathrm{Se}_{20} / /$ Recording, Storage \& Processing, 1(2), p. 19-24 (1999).
3. N.P. Eisenberg, M. Manevich, A. Arsh et al., Arrays of micro-prisms and micro-mirrors for infrared light based on $\mathrm{As}_{2} \mathrm{~S}_{3}-\mathrm{As}_{2} \mathrm{Se}_{3}$ photoresist // J. of Optoelectron. and Adv. Mater. 7(5), p. 2275 2280 (2005).

4. S.A. Kostyukevich, P.E. Shepeliavyi, N.L. Moskalenko, Investigation of the process of mastering CD on inorganic photoresists // Data Recording, Storage \& Processing, 3(4), p. 5-11 (2001).

5. V.V. Petrov, A.A. Kryuchyn, About the sensitivity of systems based on chalcogenide glassy semiconductors at high irradiation power // Kvantovaia Elektronika, 1(12), p. 2618-2620 (1974), in Russian.

6. M.T. Kostyshyn, S.A. Kostjukevich, The behavior of the light-sensitive system $\mathrm{Ag}-\mathrm{As}_{2} \mathrm{~S}_{3}$ when irradiated by laser radiation of high power // Ukr. fiz. zhurn. 26(9), p. 1561-1563 (1981), in Russian.

7. I.Z. Indutny, V.A. Danko, V.I. Minko, P.E. Shepelyavy, O.V. Bereznova, O.S. Lytvyn, Effect of photo etching in thin bowls of chalcogenide glassy semiconductors // Proc. Sci. Conf. on Semiconductor Phys. p. 99-100 (2011), in Ukrainian.

8. S.A. Kostyukevych, P.E. Shepelyavyi, N.L. Moskalenko, A.V. Vyshynskaya, S.M. Shanoylo, Y.A. Borodin, V.N. Hristin, The optimization of recording modes of master disks with As-S-Se // Data Recording, Storage \& Processing, 4(2), p. 3-10 (2002) (in Russian).

9. V.V. Petrov, A.A. Kryuchin, A.I. Britskii, A.S. Lapchuk, S.M. Shanoilo, Ways to improve the characteristics of the storage devices // Data Recording, Storage \& Processing, 12(2), p. 11-24 (2010).

10. S.A. Kostyukevich, A.N. Morozovska, P.E. Shepelyavyi et al. Recording information in thin films of chalcogenide semiconductors based on photoinduced transformations // Opt. J. 72(5), p. 76-80 (2005).

11. A.P. Prudnikov, Yu.A. Brychkov, A.P. Marychev, Integrals and Series of Elementary Functions. Moscow, Nauka, 1981 (in Russian). 